\title{
Inflammatory Bowel Disease patients requiring surgery can be treated in referral centres regardless of the COVID-19 status of the hospital: results of a multicentric European study during the first COVID-19 outbreak (COVIBD-Surg)
}

\author{
Matteo Rottoli ${ }^{1,2}$ (1) . Gianluca Pellino ${ }^{3,4} \cdot$ Marta Tanzanu $^{1,2} \cdot$ Caterina Baldi $^{5}$ - Alice Frontali ${ }^{6}$ Michele Carvello $^{7}$. \\ Caterina Foppa $^{7}$. Christos Kontovounisios ${ }^{8,9} \cdot$ Paris Tekkis $^{8,9} \cdot$ Francesco Colombo $^{10}$. Jorge Sancho-Muriel ${ }^{11}$. \\ Matteo Frasson ${ }^{11}$. Piergiorgio Danelli ${ }^{10}$. Valerio Celentano ${ }^{8,9} \cdot$ Antonino Spinelli $^{7} \cdot$ Yves Panis $^{6}$. \\ Gianluca M. Sampietro ${ }^{5}$. Gilberto Poggioli ${ }^{1,2}$
}

Received: 30 April 2021 / Accepted: 17 June 2021 / Published online: 26 June 2021

(c) Italian Society of Surgery (SIC) 2021

\begin{abstract}
Outcomes of inflammatory bowel disease (IBD) patients requiring surgery during the outbreak of Coronavirus disease 19 (COVID-19) are unknown. Aim of this study was to analyse the outcomes depending on the COVID-19 status of the centre. Patients undergoing surgery in six COVID-19 treatment and one COVID-free hospitals (five countries) during the first COVID-19 peak were included. Variables associated with risk of moderate-to-severe complications were identified using logistic regression analysis. A total of 91 patients with Crohn's disease $(54,59.3 \%)$ or ulcerative colitis $(37,40.7 \%)$, $66(72.5 \%)$ had surgery in one of the COVID-19-treatment hospitals, while $25(27.5 \%)$ in the COVID-19-free centre. More COVID-19-treatment patients required urgent surgery (48.4\% vs. $24 \%, p=0.035)$, did not discontinue biologic therapy $(15.1 \%$ vs. $0 \%, p=0.039)$, underwent surgery without a SARS-CoV-2 test $(19.7 \%$ vs. $0 \%, p=0.0033)$, and required intensive care admission $(10.6 \%$ vs. $0 \%, p=0.032)$. Three patients $(4.6 \%)$ had a SARS-CoV-2 infection postoperatively. Postoperative complications were associated with the use of steroids at surgery (Odds ratio $[\mathrm{OR}]=4.10,95 \% \mathrm{CI} 1.14-15.3, p=0.03$ ), presence of comorbidities ( $\mathrm{OR}=3.33,95 \% \mathrm{CI} 1.08-11, p=0.035)$, and Crohn's disease (vs. ulcerative colitis, OR $=3.82$, $95 \%$ CI 1.14-15.4, $p=0.028$ ). IBD patients can undergo surgery regardless of the COVID-19 status of the referral centre. The risk of SARS-CoV-2 infection should be taken into account.
\end{abstract}

Keywords Inflammatory bowel disease $\cdot$ Surgery $\cdot$ COVID-19

Matteo Rottoli

matteo.rottoli2@unibo.it ${ }^{1}$

Surgery of the Alimentary Tract,

IRCCS Azienda Ospedaliero-Universitaria di Bologna,

Bologna, Italy

2 Department of Medical and Surgical Sciences, Alma Mater Studiorum University of Bologna, Via Massarenti 9, 40138 Bologna, Italy

3 Department of Advanced Medical and Surgical Sciences, Università degli Studi della Campania "Luigi Vanvitelli", Naples, Italy

4 Colorectal Surgery, Vall d'Hebron University Hospital, Barcelona, Spain

5 Divisione di Chirurgia Generale e Epato-Bilio-Pancreatica, Ospedale di Rho-ASST Rhodense, Rho, Milan, Italy
6 Department of Colorectal Surgery, Pôle des Maladies de l'Appareil Digestif (PMAD), Beaujon Hospital, Assistance Publique-Hôpitaux de Paris (AP-HP), University Denis Diderot (Paris VII), Clichy Cedex, France

7 Colon and Rectal Surgery Division, Department of Biomedical Science, Humanitas Clinical and Research Center, Humanitas University, Rozzano, MI, Italy

8 Department of Surgery and Cancer, Imperial College, London, UK

9 Chelsea and Westminster Hospital NHS Foundation Trust, London, UK

10 Department of General Surgery, Department of Biomedical and Clinical Sciences "L. Sacco", University of Milan, ASST Fatebenefratelli Sacco, Milan, Italy

11 Colorectal Unit, Hospital Universitario y Politecnico La Fe, University of Valencia, Valencia, Spain 


\section{Introduction}

The SARS-CoV-2 (severe acute respiratory syndrome coronavirus 2) pandemic hit Europe notably during the first semester of 2020. Italy and, subsequently, other European countries witnessed an exponential increase in cases and deaths associated with the uncontrolled spread of the infection outside of China. [1, 2] The need of increased capacity for Coronavirus disease 19 (COVID-19) patients forced many hospitals to postpone or cancel elective surgery, including elective procedures for inflammatory bowel disease (IBD), despite the higher risk of these patients of requiring urgent surgery.

While it has been suggested that major surgery should be performed in COVID-19-free hospitals to reduce the perioperative risk of infection and the mortality rate [3, 4], this possibility was not always possible in the case of IBD patients since their treatment is often centralised in only a few referral centres, the majority of which were reorganised into COVID-19 hubs during the first outbreak of the pandemic. Therefore, it was unclear whether the perioperative risks of IBD patients requiring surgery increased when surgery was carried out in COVID-19-treatment hospitals, due to either the unavoidable paucity of resources or the risk of SARS-CoV-2 infection. Specific analysis of the outcomes of IBD patients was of particular importance since a significant proportion of these patients undergo immunomodulatory therapy which could affect the perioperative outcomes and increase the risk of infection [5].

The aim of this study was, therefore, to analyse the characteristics and the perioperative outcomes of IBD patients undergoing surgery during the "healthcare lockdown" due to the first peak of the COVID-19 outbreak in seven European referral centres which were dedicated to the care of COVID-19 patients, and to compare them to the outcomes of the patients who underwent surgery in the only IBD tertiary centre which was located in a COVID-free hospital.

\section{Methods}

This was a retrospective study involving all consecutive patients who underwent surgery for IBD in seven referral centres across five European countries between 9 March and 30 June 2020. Four centres were located in Italy (Sant'Orsola Hospital, Alma Mater Studiorum University of Bologna; Humanitas Research Hospital, Rozzano, Milan; Luigi Sacco University Hospital, Milan; Rho Memorial Hospital, Rho, Milan), one in France (Beaujon Hospital, Paris), one in Spain (Hospital Universitario y Politecnico La Fe,
University of Valencia, Valencia) and one in the United Kingdom (Chelsea and Westminster Hospital NHS Foundation Trust, London).

To analyse the potential correlation between the restructuring of the clinical resources due to the COVID-19 pandemic and the outcomes of patient undergoing IBD surgery, the study considered only the period of time during which the rate of the elective procedures was reduced to a minimum. This period, which varied among the European countries depending on the different distribution of the SARSCoV-2 infection in the population, was: 10 March-4 May in Italy, 14 March-11 May in Spain, 1 April-30 June in the United Kingdom, and 9 March-10 May in France. During the study period, six hospitals were restructured to become COVID-19-treatment hubs, although a COVID-19-free surgical pathway was established in all centres, to reduce the risk of cross-infection of patients undergoing surgery. One centre (Rho Memorial Hospital, Rho, Milan, Italy) was located in a COVID-free hospital, having, therefore, access to a similar number of resources as before the pandemic, in particular in terms of operating theatre, intensive care beds, and personnel availability.

All the patients who were admitted to hospital for surgery underwent a nasopharyngeal swab to exclude a preoperative diagnosis of SARS-CoV-2 infection, unless the emergency of the procedure required otherwise. In all COVID-19 treatment centres, a COVID-19-free pathway was established from the surgical ward to the operative theatre, and viceversa. The patients received surgery in a COVID-19-free operative room, and the whole surgical ward did not admit any COVID-19-positive or suspected patient.

Demographic characteristics, preoperative medical treatment, perioperative variables, the perioperative state of SARS-CoV-2 infection, and complications within 30 days were recorded in a dedicated database using REDCap (Research Electronic Data Capture) tools at Alma Mater Studiorum University of Bologna [6]. The study was approved by the Ethics Committee of the coordinating centre (Sant'Orsola Hospital, Bologna) and carried out in the other centres according to local regulations.

\section{Statistical analysis}

The continuous variables were expressed as mean ( \pm standard deviation [SD]) while the categorical variables were presented as number (\%). The differences in the categorical and the continuous variables were evaluated using the Chi-squared test and the $t$ test, respectively. All $p$ values refer to two-tailed tests of significance. The potential independent predictors of moderate-to-severe postoperative 
Table 1 Demographic variables and disease characteristics of the patients enrolled in the study

\begin{tabular}{|c|c|c|}
\hline & $N$ or mean & $\%$ or $\pm \mathrm{SD}$ \\
\hline Total number of patients & 91 & \\
\hline Mean age (years) & 43 & \pm 17.1 \\
\hline Male gender & 59 & $64.8 \%$ \\
\hline Mean BMI $\left(\mathrm{kg} / \mathrm{m}^{2}\right)$ & 22.5 & \pm 3.5 \\
\hline Any comorbidity & 38 & $41.8 \%$ \\
\hline Extra-intestinal manifestation & 16 & $17.6 \%$ \\
\hline \multicolumn{3}{|l|}{ Diagnosis } \\
\hline Ulcerative colitis & 37 & $40.7 \%$ \\
\hline Crohn's disease & 54 & $59.3 \%$ \\
\hline Preoperative diagnosis of cancer & 5 & $5.5 \%$ \\
\hline \multicolumn{3}{|l|}{ Medication } \\
\hline Steroids at time of surgery & 18 & $19.8 \%$ \\
\hline Azathioprine at time of surgery & 5 & $5.5 \%$ \\
\hline History of exposure to biologics & 49 & $55.1 \%$ \\
\hline Biologics at time of surgery & 14 & $15.4 \%$ \\
\hline No discontinuation of biologics at surgery & 10 & $11.0 \%$ \\
\hline \multicolumn{3}{|l|}{ Ulcerative colitis (37 patients)—details } \\
\hline Pancolitis & 18 & $48.6 \%$ \\
\hline Moderate-to-severe disease activity & 27 & $73 \%$ \\
\hline Strictures & 4 & $10.8 \%$ \\
\hline \multicolumn{3}{|l|}{ Crohn's disease (54 patients)—details } \\
\hline L1 Montreal classification & 22 & $40.7 \%$ \\
\hline L2 Montreal classification & 8 & $14.8 \%$ \\
\hline L3 Montreal classification & 24 & $44.4 \%$ \\
\hline Upper gastrointestinal localization & 2 & $3.7 \%$ \\
\hline Fistulizing behavior & 27 & $50 \%$ \\
\hline Perineal disease & 19 & $35.2 \%$ \\
\hline
\end{tabular}

$N$ : number, $S D$ standard deviation, $B M I$ body mass index

complications (defined as grade 2 or higher according to the Clavien-Dindo classification) were evaluated using logistic regression analysis (using the data obtained at 30 days of follow-up). Considering the presence of a large number of regressors and the high risk of over-fitting due to the small number of events, the variables to be included in the models using the least absolute shrinkage and selection operator (LASSO) method were selected. A $p$ value $<0.05$ was considered significant. The statistical analyses were carried out using Stata/SE version 16 (StataCorp LLC, College Station, Texas, USA).

\section{Results}

A total of 91 patients (59 males, 64.8\%) underwent surgery for Crohn's disease (CD; 54, 59.3\%) and ulcerative colitis (UC; 37, 40.7\%) during the study period. No patient was excluded from the study. Five patients $(5.5 \%)$ had a preoperative diagnosis of cancer. The details of the population of patients enrolled in the study are reported in Table 1. A total of 66 patients (72.5\%) had surgery in one of the 6 COVID-19-treatment hospitals while 25 cases (27.5\%) were treated in the COVID-19-free centre. The comparison between these two populations of patients is reported in Table 2. The distribution of CD (59.1\% vs. $60 \%)$ and UC ( $40.9 \%$ vs. $40 \%, p=0.93)$ diagnoses, the use of steroids at the time of surgery $(19.7 \%$ vs. $20 \%, p=0.97)$ and the preoperative exposure to biologics $(59.1 \%$ vs. $40 \%, p=0.10)$ did not differ between the groups.

A significantly higher percentage of patients who were admitted to COVID-19-treatment hospitals underwent urgent surgery $(48.4 \%$ vs. $24 \%, p=0.035)$, which resulted in a greater proportion of patients who underwent surgery without a preoperative test for SARS-CoV-2 infection (19.7\% vs. $0 \%, p=0.0033$ ), without proper discontinuation of the biologic therapy $(15.1 \%$ vs. $0 \%, p=0.039)$, and who were at greater risk of having a diverting ileostomy after a resection and anastomosis ( $50 \%$ vs. $17.7 \%, p=0.018)$. Since the present study was retrospective, it was not clear whether the higher rate of diverting ileostomy was due to the emergency setting or the call for extra-caution given the context. Three of the patients (4.6\%) who had surgery in COVID-19-treatment hospitals were diagnosed with a SARS-CoV-2 infection during the postoperative course. No patient required any escalation of treatment due to the infection.

Table 3 reports the details of the surgical indications and the procedures performed on the patients enrolled in the study.

At the logistic regression analysis (Table 4), the only variables associated with the risk of postoperative complications (classified as Clavien-Dindo 2 and higher) were: the use of steroids at the time of surgery (Odds ratio [OR] 4.10, 95\% CI $1.14-15.3, p=0.03$ ), the report of comorbidities (OR 3.33, 95\% CI 1.08-11, $p=0.035$ ), and the diagnosis of Crohn's disease (vs. ulcerative colitis; OR 3.82, 95\% CI 1.14-15.4, $p=0.028$ ), while having surgery in a COVID-19-treatment hospital was not associated with a higher risk of complications (OR 2.06, 95\% CI 0.54-10.4, $p=0.3$ ).

\section{Discussion}

Patients affected by IBD were identified as a population at potential higher risk of severe illness in case of SARS$\mathrm{CoV}-2$ infection, especially taking into account that many of them undergo immunomodulatory therapy, and the guidelines focused on maintaining the remission of disease and postponing any non-urgent surgery to avoid unnecessary hospital admissions [7-9]. Nonetheless, delaying the time of surgery might have increased the risk of complications and poor outcomes of IBD patients who required surgery 
Table 2 Comparison of preoperative characteristics and outcomes between the patients requiring IBD surgery and admitted to the COVID-19treatment and COVID-19-free hospitals

\begin{tabular}{|c|c|c|c|}
\hline & $\begin{array}{l}\text { COVID-19-treatment } \\
\text { hospital }\end{array}$ & $\begin{array}{l}\text { COVID-19-free } \\
\text { hospital }\end{array}$ & $p$ value \\
\hline Number of patients & $66(72.5 \%)$ & $25(27.5 \%)$ & \\
\hline Age at surgery (years) & $43.7 \pm 2.1$ & $43.8 \pm 3.4$ & 0.98 \\
\hline Age at diagnosis & $28.7 \pm 2.1$ & $35.9 \pm 3.2$ & 0.031 \\
\hline Male gender & $41(62.1 \%)$ & $18(72 \%)$ & 0.38 \\
\hline BMI $\left(\mathrm{kg} / \mathrm{m}^{2}\right)$ & $22.5 \pm 3.5$ & $22.4 \pm 3.6$ & 0.91 \\
\hline Any comorbidity & $31(47 \%)$ & $7(28 \%)$ & 0.10 \\
\hline Extra-intestinal manifestation & $14(21.2 \%)$ & $2(8 \%)$ & 0.13 \\
\hline Referred from other centre & $10(15.2 \%)$ & $20(83.3 \%)$ & $<0.0001$ \\
\hline \multicolumn{4}{|l|}{ Diagnosis } \\
\hline Ulcerative colitis & $27(40.9 \%)$ & $10(40 \%)$ & 0.93 \\
\hline Crohn's disease & $39(59.1 \%)$ & $15(60)$ & \\
\hline Preoperative diagnosis of cancer & $3(4.6 \%)$ & $2(8 \%)$ & 0.52 \\
\hline \multicolumn{4}{|l|}{ Medication } \\
\hline Steroids at time of surgery & $13(19.7 \%)$ & $5(20 \%)$ & 0.97 \\
\hline Azathioprine at time of surgery & $4(6.1 \%)$ & $1(4 \%)$ & 0.70 \\
\hline History of exposure to biologics & $39(59.1 \%)$ & $10(40 \%)$ & 0.10 \\
\hline No discontinuation of biologics for the surgery & $10(15.1 \%)$ & $0(0 \%)$ & 0.039 \\
\hline \multicolumn{4}{|l|}{ Ulcerative colitis ( 37 patients)_details } \\
\hline Strictures & $4(15.4 \%)$ & $0(0 \%)$ & 0.09 \\
\hline \multicolumn{4}{|l|}{ Crohn's disease (54 patients)—details } \\
\hline Fistulising behavior & $18(46.1 \%)$ & $9(60 \%)$ & 0.44 \\
\hline Perineal disease & $16(24.2 \%)$ & $3(12 \%)$ & 0.20 \\
\hline Patients transferred from other wards & $12(18.2 \%)$ & $5(20 \%)$ & 0.84 \\
\hline \multicolumn{4}{|l|}{ Preoperative COVID-19 diagnosis } \\
\hline Negative at preoperative swabs & $53(80.3 \%)$ & $24(96 \%)$ & 0.0033 \\
\hline History of positivity, preoperatively negative & $0(0 \%)$ & $1(4 \%)$ & \\
\hline Preoperative test not performed due to emergency & $13(19.7 \%)$ & $0(0 \%)$ & \\
\hline Urgent or expedited surgery & $32(48.4 \%)$ & $6(24 \%)$ & 0.035 \\
\hline \multicolumn{4}{|l|}{ Surgical indication } \\
\hline Confirmed or suspected cancer, dysplasia & $7(10.6 \%)$ & $2(8 \%)$ & 0.92 \\
\hline Non-septic complications & $29(43.9 \%)$ & $11(44 \%)$ & \\
\hline Septic complications & $17(25.8 \%)$ & $8(32 \%)$ & \\
\hline Complications of existing stoma & $13(19.7 \%)$ & $4(16 \%)$ & \\
\hline ASA score $>2$ & $6(9.1 \%)$ & $1(4 \%)$ & 0.41 \\
\hline $\begin{array}{l}\text { Diverting ileostomy (in case of resection and anastomosis }=57 \\
\text { cases) }\end{array}$ & $20(50 \%)$ & $3(17.7 \%)$ & 0.018 \\
\hline Intraoperative medical or surgical complications & $1(1.52 \%)$ & $0(0 \%)$ & 0.53 \\
\hline Scheduled postoperative ICU & $7(10.6 \%)$ & $0(0 \%)$ & 0.032 \\
\hline Postoperative medical complications & $10(15.1 \%)$ & $3(12 \%)$ & 0.70 \\
\hline Postoperative surgical complications & $9(13.6 \%)$ & $2(8 \%)$ & 0.46 \\
\hline Reoperations & $0(0 \%)$ & $2(8 \%)$ & 0.021 \\
\hline Clavien-Dindo classification & Out of 16 & Out of 4 & 0.037 \\
\hline Grade I & $0(0 \%)$ & $1(25 \%)$ & \\
\hline Grade II & $12(75 \%)$ & $1(25 \%)$ & \\
\hline Grade IIIa & $3(18.8 \%)$ & $0(0 \%)$ & \\
\hline Grade IIIb & $1(6.25 \%)$ & $1(25 \%)$ & \\
\hline Grade IV & $0(0 \%)$ & $1(25 \%)$ & \\
\hline 30-day SARS-CoV-2 infection rate & $3(4.6 \%)$ & $0(0 \%)$ & 0.16 \\
\hline Length of stay (days) & $8.7 \pm 9$ & $11.3 \pm 9.1$ & 0.23 \\
\hline Cancer confirmed at histology & $3(4.6 \%)$ & $3(12 \%)$ & 0.21 \\
\hline 30-day mortality & $0(0 \%)$ & $0(0 \%)$ & - \\
\hline
\end{tabular}

$I B D$ inflammatory bowel disease, $B M I$ body mass index, $I C U$ intensive care unit, $A S A$ American Society of Anesthesiologists 
Table 3 Details regarding the indication for surgery and the surgical procedures performed on the patients enrolled in the study
COVID-19 treating hospi-

\section{tal (66)}

COVID-19-

free hospital

(25)

\begin{tabular}{lll} 
Indication for surgery & & \\
Severe dysplasia or cancer & 7 & 2 \\
Disease unresponsive to medical treatment & 12 & 2 \\
CD: stenosis & 13 & 7 \\
CD: enteric fistula & 13 & 7 \\
UC: Toxic megacolon & 1 & 0 \\
Bleeding & 2 & 2 \\
Bowel perforation & 2 & 1 \\
Bowel obstruction & 1 & 0 \\
Perianal sepsis & 2 & 0 \\
Stoma complications (including high output) & 13 & 4 \\
Surgical procedure & & \\
Subototal colectomy and end ileostomy & 10 & 5 \\
Subototal colectomy and ileorectal anastomosis & 1 & 0 \\
Proctocolectomy and IPAA formation & 4 & 0 \\
Proctocolectomy and abdomino-perineal resection & 2 & 0 \\
Proctectomy and IPAA formation & 8 & 3 \\
Proctectomy and abdomino-perineal resection & 1 & 0 \\
Ileocecal resection & 13 & 12 \\
Small bowel resection & 7 & 0 \\
Segmental colonic resection & 3 & 3 \\
Ileostomy formation & 1 & 0 \\
Perianal surgery & 2 & 0 \\
Reversal of stoma & 10 & 2 \\
Other & 4 & 0 \\
Laparoscopic procedures & 31 & \\
\hline & & \\
\hline
\end{tabular}

$C D$ Crohn's disease, $U C$ ulcerative colitis, IPAA ileal pouch-anal anastomosis

Table 4 Logistic regression analysis of the association between the independent variables and the risk of postoperative complications, classified as Clavien-Dindo grade 2 and higher

\begin{tabular}{llll}
\hline Variable & Odds ratio & $95 \%$ CI & $p$ value \\
\hline COVID-19-treatment hospital (vs. COVID-19 free) & 2.06 & $0.54-10.4$ & 0.30 \\
Steroid therapy at time of surgery (vs. no steroids) & 4.10 & $1.14-15.3$ & 0.030 \\
Any comorbidity (vs. no comorbidities) & 3.33 & $1.08-11$ & 0.035 \\
Diagnosis of Crohn's (vs. ulcerative colitis) & 3.82 & $1.14-15.4$ & 0.028 \\
\hline
\end{tabular}

[10]. In real life, physicians and surgeons had to deal with patients with IBD potentially requiring surgery on a caseby-case basis, taking into account the unprecedented context in terms of risks and lack of resources. Apart from the obvious conditions which required immediate surgical treatment, other less-urgent situations had to be carefully assessed. For instance, in the present series, a total of 12 patients underwent a procedure which included the closure of a stoma (reversal of ileostomy, construction of an ileo-colonic anastomosis) and 11 underwent a second-stage pouch formation; seventeen of them reported a high stoma output which required multiple hospital admissions. The need for surgery had to be balanced with the possible risk of infection and poor outcomes in these often frail patients. During the first outbreak of the COVID-19 pandemic, little was known about whether IBD patients were at higher risk of contracting severe COVID-19, and whether there was a correlation between immunosuppressive and biological therapy, and the coronavirus infection [11]. Previous evidence regarding a higher risk of viral infections in IBD patients on 
thiopurine therapy [12], and a greater tissue concentration of angiotensin-converting enzyme 2 in patients with active intestinal inflammation [13], contributed to raising concerns regarding the threat of severe illness in IBD patients in the case of SARS-CoV-2 infection. Only in subsequent months did additional population studies show that patients affected by IBD had a risk of contracting coronavirus infection similar to that of the general population $[14,15]$, regardless of the biological therapy [16-18]. However, it is still not known whether the outcomes of surgery performed on IBD patients during the period of highest pressure to the healthcare system were affected by the environment of a COVID19-treatment hospital, and whether IBD surgery should be performed only in COVID-19-free facilities.

To the authors' knowledge, to date, no other large series of IBD surgical patients have been described in details [19, 20]. The present study showed that the IBD patients admitted in COVID-19 treatment and COVID-19-free hospitals were comparable in terms of demographic characteristics, preoperative medications and histological diagnosis. However, patients who had surgery in a COVID-19 treatment hospital had a greater chance of undergoing urgent surgery ( $48.4 \%$ vs. $24 \%, p=0.035$ ), probably due to the need for the IBD patients to be triaged to surgery based on the degree of urgency, to properly manage the limited resources. The higher proportion of urgent procedures, as well as the tendency towards an over-cautious approach, could explain the higher rate of diverting ileostomy after a resection and anastomosis in these centres (50\% vs. $17.7 \%, p=0.018$ ).

The Intercollegiate General Surgery Guidance on COVID-19, which was published on 25th March 2020, proposed to consider stoma formation rather than primary anastomosis in case of resection, to reduce the risk of postoperative complications and longer hospital stay [21]. A correlation between mortality and anastomotic leak after colorectal surgery was also found by the COVIDSurg Collaborative in their study, leading the authors to suggest a low threshold for diverting ileostomy [4]. The present study showed that the formation of an anastomosis after resection was safe in IBD patients, as this variable was not associated with a higher risk of postoperative complications in our analysis. In fact, the only significant risk factors for the onset of a moderate-to-severe complication (classified as Clavien-Dindo >1) were on-going steroid therapy at the time of surgery, a diagnosis of Crohn's disease, and the presence of comorbidities, while the COVID-19 hospital setting did not correlate with the risk of complications. Nevertheless, three patients $(4.6 \%)$ who had surgery in a COVID-19-treatment centre developed a coronavirus infection, although none of them had severe outcomes from the infection or required admission to intensive care.
The present study had a few limitations. First, there was no certain definition of the parameters to define the study period among the different countries. The principal investigators in each centre were asked to choose the period during which the redistribution of the resources from elective surgery was the gravest. Second, the availability of clinical resources was different from hospital to hospital, depending on the location. In some centres (in particular, those located in the Lombardy region of Italy), redistribution of the hospital capacity to the care of COVID-19 patients was almost total while, in other centres, elective surgery was greatly reduced but not completely cancelled. Third, the limited number of cases and events decreased the accuracy of the regression analysis. Nevertheless, the present study reported the largest series of patients to date who underwent surgery for IBD during the first wave of the COVID-19 pandemic, and showed that the COVID-19 hospital setting did not affect the outcomes, suggesting that the centralisation of IBD surgery in referral centres might have contributed to maintaining a low rate of postoperative complications, despite the fact that a SARS-CoV-2 infection in the postoperative period represented a concrete risk in IBD patients undergoing surgery in COVID-19-treatment hospitals. In particular, these findings also highlight the lack of a standardised pathway for the management of IBD patients requiring either emergency or elective surgical treatment during the pandemic which is still on-going and, in the authors' opinion, support the creation of hubs dedicated to the care of IBD patients, similar to what has been done with cancertreatment hubs [22], to ensure that optimal treatment is delivered also to patients affected by IBD.

\section{Conclusions}

Although the unavoidable risk of in-hospital SARS-CoV-2 infection has to be taken into account, the present study showed that IBD patients can undergo surgery with good outcomes regardless of the COVID-19 status of the referral centre, even during the worst peak of the COVID-19 pandemic.

Supplementary Information The online version contains supplementary material available at https://doi.org/10.1007/s13304-021-01119-y.

Author contributions All authors contributed to the study conception and design. Material preparation, data collection and analysis were performed by Matteo Rottoli, Gianluca Pellino, Marta Tanzanu, Caterina Baldi, Alice Frontali, Michele Carvello, Caterina Foppa, Christos Kontovounisios, Francesco Colombo, and Jorge Sancho-Muriel. The first draft of the manuscript was written by Matteo Rottoli. Paris Tekkis, Matteo Frasson, Piergiorgio Danelli, Valerio Celentano, Antonino Spinelli, Yves Panis, Gianluca M Sampietro, and Gilberto Poggioli 
commented on the manuscript and contributed to the final version, which was read and approved by alla authors.

Funding No specific funding was received for the present study.

Data availability The datasets generated during and/or analysed during the current study are available from the corresponding author on reasonable request.

\section{Declarations}

Conflict of interest The authors declare that they have no conflict of interest.

Research involving human participants The research was conducted according the the ethical standards. The study was approved by the ethical committee of the leading centre (Area Vasta Emilia CentroAVEC) and carried out to the participating centres according to the local regulations.

Informed consent An informed consent was obtained by the patients when possible.

\section{References}

1. Onder G, Rezza G, Brusaferro S (2020) Case-fatality rate and characteristics of patients dying in relation to COVID-19 in Italy. JAMA 323:1775-1776

2. World Health Organization Coronavirus disease 2019 (COVID19): situation report-67. March 31, 2020. https://www.who.int/ docs/default-source/coronaviruse/situation-reports/20200407sitrep-78-covid-19.pdf?sfvrsn=bc43e1b_2. Accessed 30 Jan 2021

3. COVIDSurg Collaborative (2020) Mortality and pulmonary complications in patients undergoing surgery with perioperative SARS-CoV-2 infection: an international cohort study. Lancet 396:27-38

4. COVIDSurg Collaborative (2020) Outcomes from elective colorectal cancer surgery during the SARS-CoV-2 pandemic. Colorectal Dis. https://doi.org/10.1111/codi.15431

5. Tinsley A, Navabi S, Williams ED, Liu G, Kong L, Coates MD, Clarke K (2019) Increased risk of influenza and influenza-related complications among 140,480 patients with inflammatory bowel disease. Inflamm Bowel Dis 25:369-376

6. Harris PA, Taylor R, Thielke R, Payne J, Gonzalez N, Conde JG (2009) Research electronic data capture (REDCap) — a metadatadriven methodology and workflow process for providing translational research informatics support. J Biomed Inform 42:377-381

7. Magro F, Rahier JF, Abreu C, MacMahon E, Hart A, van der Woude CJ, Gordon H, Adamina M, Viget N, Vavricka S, Kucharzik T, Leone S, Siegmund B, Danese S, Peyrin-Biroulet L (2020) Inflammatory bowel disease management during the COVID-19 outbreak: the ten do's and don'ts from the ECCO-COVID taskforce. J Crohns Colitis 14(Suppl_3):S798-S806

8. Kennedy NA, Jones GR, Lamb CA, Appleby R, Arnott I, Beattie RM, Bloom S, Brooks AJ, Cooney R, Dart RJ, Edwards C, Fraser A, Gaya DR, Ghosh S, Greveson K, Hansen R, Hart A, Hawthorne AB, Hayee B, Limdi JK, Murray CD, Parkes GC, Parkes M, Patel K, Pollok RC, Powell N, Probert CS, Raine T, Sebastian S, Selinger C, Smith PJ, Stansfield C, Younge L, Lindsay JO, Irving PM, Lees CW (2020) British Society of Gastroenterology guidance for management of inflammatory bowel disease during the COVID-19 pandemic. Gut 6:984-990

9. Remzi FH, Panis Y, Spinelli A, Kotze PG, Mantzaris G, Söderholm JD, d'Hoore A, Bemelman WA, Yamamoto T, Pemberton JH, Tiret E, Øresland T, Fleshner P (2020) International organization for the study of IBD recommendations for surgery in patients with IBD during the coronavirus disease 2019 pandemic. Dis Colon Rectum 63:870-873

10. Allez M, Fleshner P, Gearry R, Lakatos PL, Rubin DT (2020) Care of the patient with IBD requiring hospitalisation during the COVID-19 pandemic. J Crohns Colitis 14(Suppl_3):S774-S779. https://doi.org/10.1093/ecco-jcc/jjaa150

11. D'Amico F, Peyrin-Biroulet L, Danese S (2020) Inflammatory bowel diseases and COVID-19: the invisible enemy. Gastroenterology 158:2302-2304

12. Wisniewski A, Kirchgesner J, Seksik P, Landman C, Bourrier A, Nion-Larmurier I, Marteau P, Cosnes J, Sokol H, Beaugerie L, the Saint-Antoine IBD network (2020) Increased incidence of systemic serious viral infections in patients with inflammatory bowel disease associates with active disease and use of thiopurines. United Eur Gastroenterol J 8:303-313

13. Garg M, Royce SG, Tikellis C, Shallue C, Batu D, Velkoska E, Burrell LM, Patel SK, Beswick L, Jackson A, Britto K, Lukies M, Sluka P, Wardan H, Hirokawa Y, Tan CW, Faux M, Burgess AW, Hosking P, Monagle S, Thomas M, Gibson PR, Lubel J (2020) Imbalance of the renin-angiotensin system may contribute to inflammation and fibrosis in IBD: a novel therapeutic target? Gut 69:841-851

14. Taxonera C, Sagastagoitia I, Alba C, Mañas N, Olivares D, Rey E (2020) 2019 Novel coronavirus disease (COVID-19) in patients with inflammatory bowel diseases. Aliment Pharmacol Ther $52: 276-283$

15. Allocca M, Fiorino G, Zallot C, Furfaro F, Gilardi D, Radice S, Danese S, Peyrin-Biroulet L (2020) Incidence and patterns of COVID-19 among inflammatory bowel disease patients from the Nancy and Milan cohorts. Clin Gastroenterol Hepatol 18:2134-2135

16. Bezzio C, Saibeni S, Variola A, Allocca M, Massari A, Gerardi V, Casini V, Ricci C, Zingone F, Amato A, Caprioli F, Lenti MV, Viganò C, Ascolani M, Bossa F, Castiglione F, Cortelezzi C, Grossi L, Milla M, Morganti D, Pastorelli L, Ribaldone DG, Sartini A, Soriano A, Manes G, Danese S, Fantini MC, Armuzzi A, Daperno M, Fiorino G, Italian Group for the Study of Inflammatory Bowel Disease (IG-IBD) (2020) Outcomes of COVID-19 in 79 patients with IBD in Italy: an IG-IBD study. Gut 69:1213-1217

17. Brenner EJ, Ungaro RC, Gearry RB, Kaplan GG, Kissous-Hunt M, Lewis JD, Ng SC, Rahier JF, Reinisch W, Ruemmele FM, Steinwurz F, Underwood FE, Zhang X, Colombel JF, Kappelman MD (2020) Corticosteroids, but not TNF antagonists, are associated with adverse COVID-19 outcomes in patients with inflammatory bowel diseases: results from an international registry. Gastroenterology 159:481-491

18. Rizzello F, Calabrese C, Salice M, Calandrini L, Privitera H, Melotti L, Peruzzi G, Dussias N, Belluzzi A, Scaioli E, Decorato A, Siniscalchi A, Filippone E, Laureti S, Rottoli M, Poggioli G, Gionchetti P (2020) COVID-19 in IBD: The experience of a single tertiary IBD center. Dig Liver Dis 53:271-276

19. Lan N, Zhi M, Chen Y, Wu X, Lan P, Kiran RP, Shen B (2020) Experience of hospital admission and surgery during the COVID19 pandemic: a survey of IBD patients. J Gastrointest Surg 3:1-5

20. Botti F, Mazza S, Costa S, Farina E, Baldari L, Prati M (2020) Urgent surgery for inflammatory bowel diseases during the COVID-19 outbreak in a high incidence area-experience from a high-volume centre. Dig Liver Dis 52:1241-1243 
21. Urgent Intercollegiate General Surgery Guidance on COVID19, posted on 25 March 2020. https://www.acpgbi.org.uk/news/ urgent-intercollegiate-general-surgery-guidance-on-covid-19/. Accessed 1 Feb 2021

22. United Kingdom National Health System: advice on maintaining cancer treatment during the COVID-19 response, 30 March 2020. https://www.bopa.org.uk/wp-content/uploads/2020/03/C0119-_
Maintaining-cancer-services-_-letter-to-trusts.pdf. Accessed 1 Feb 2021

Publisher's Note Springer Nature remains neutral with regard to jurisdictional claims in published maps and institutional affiliations. 